\title{
Development of a pediatric transfusion medicine teaching curriculum for pediatric anesthesiology fellows
}

\begin{abstract}
Context: Formal training in pediatric transfusion medicine is often centered on education of Clinical Pathology and Hematology/Oncology Fellows. The Children's Hospital of Philadelphia (CHOP) accepts nine fellows annually into a Pediatric Anesthesiology and Critical Care Medicine (PACCM) Fellowship Training Program. Training requirements include the appropriate ordering and utilization of blood products for pediatric patients, which many fellows have limited prior experience.
\end{abstract}

Objective: To design, implement, and review a teaching curriculum for PACCM Fellows to introduce these physician trainees to the unique features of pediatric TM and hospital specific policies.

Design: Starting in 2006, two fellows per session were assigned a full day in the blood bank. The schedule included "hands-on" learning and lectures. Each fellow received lecture handouts and TM literature. They were asked to submit a pre- and post-test, an evaluation form and give oral feedback. Additional lectures were given during morning conferences as part of their overall educational training during their fellowship.

Results: Twenty-eight fellows have completed this rotation to date of this curriculum review period. Most fellows demonstrated knowledge of the definition of clinically significant antibodies and proper labeling of blood samples. Fewer fellows demonstrated knowledge of $\mathrm{CHOP}$ specific policies prior to the rotation. Twenty-three written evaluations with ratings of outstanding to good were received. As a result of the teaching curriculum, there is less miscommunication, fewer customer complaints and occurrence reports (safety net reports) documented involving TM and PACCM departments. There has also been a steady overall improvement in client satisfaction.

Conclusion: We report the development of a pediatric TM curriculum designed for anesthesiology fellows. The curriculum will continue to evolve as additional feedback is received. We will continue to monitor the curriculum in terms of an improvement in pre and post testing results, overall physician satisfaction and safety net reports.
Volume I Issue I - 2015

\author{
Yvette C Tanhehco,' Carmelita Moultrie- \\ Savage, ${ }^{2}$ Pamala Blair, ${ }^{2}$ David Friedman, ${ }^{2}$ \\ Ronald S Litman, ${ }^{3}$ Alan Jay Schwartz, ${ }^{3}$ \\ Deborah A Sesok-Pizzini \\ 'Department of Pathology and Cell Biology, Columbia \\ University, United States \\ 2Department of Pathology and Laboratory Medicine, The \\ Children's Hospital of Philadelphia, United States \\ ${ }^{3}$ Department of Anesthesiology and Critical Care Medicine, The \\ Children's Hospital of Philadelphia, United States
}

\begin{abstract}
Correspondence: Deborah A. Sesok-Pizzini, Department of Pathology and Laboratory Medicine Blood Bank and Transfusion Medicine, Children's Hospital of Philadelphia, 34th Street and Civic Center Boulevard, Philadelphia, United states, Tel 215-5900369, Fax 215-590-2171, Email pizzini@email.chop.edu
\end{abstract}

Received: April 7, 2015 | Published: May 25, 2015

\section{Introduction}

Over 20,000 units of blood products (packed red blood cell, plasma, platelet and cryoprecipitate) are utilized each year at CHOP. Of these, $25 \%$ are ordered by the PACCM department. Training on the appropriate ordering and utilization of blood products for pediatric patients in the intensive care unit (ICU) and the operating room (OR) is not routinely given to residents and fellows. As a result, an average of $5 \%$ of products is estimated to be wasted each year.

Knowledge of TM by residents, fellows and attendings in various clinical departments needs improvement. ${ }^{1-5}$ Eisenstaedt et $\mathrm{al}^{2}$ conducted a survey among resident physicians at nine academic medical centers to assess their perceptions of their knowledge of $\mathrm{TM}$, their confidence in using various blood bank resources, and their ratings of the contribution of various learning resources to their knowledge of TM. Nearly $90 \%$ of the residents who took the survey were in an internal medicine, surgery or anesthesiology residency program. Respondents placed a high value on the relevance of TM to patient care; however, they rated their knowledge and learning opportunities as only moderate.
TM and anesthesiology are closely related fields that share common interests. An anesthesiologist should not only have an understanding of the basic science of TM, but also the more practical clinical aspects of TM. It is not enough for anesthesiologists to know about immunology, hemoglobin levels, blood coagulation and the hemodynamics of circulation; they must also be familiar with turnaround times for pretransfusion testing and manufacturing requests, autologous blood transfusion, indications for the appropriate use of blood components and transfusion reactions. ${ }^{6}$ In order to maximize patient safety, anesthesiologists must have a clear understanding of the safe use of blood products during surgery and in the ICU.

Blood products such as whole blood, packed red blood cells $(\mathrm{pRBC})$, plasma and platelets are routinely used in the OR and ICUs for hemodynamic and coagulation support. The type and quantity of blood products needed are dependent upon the clinical history of the patient as well as the type of surgery being performed. Utilization of blood products in the OR or ICU may not always be anticipated in advance so urgent orders for blood products are often placed with the blood bank. It is not uncommon for complaints to be received in the blood bank regarding the length of time it takes for a blood product 
to be issued. A lack of understanding of the processes and procedures that need to be performed prior to the release of a blood product often results in unmet expectations, communication breakdowns and conflicts between the TM and PACCM departments.

Many anesthesiology fellows have limited experience with pediatric TM. Although the major concepts are similar between pediatric and adult TM, there are some additional considerations in the preparation and administration of blood products to the pediatric population due to the child's smaller volume and more immature immune system. At CHOP, a need was identified by both the TM and PACCM departments to educate their fellows on the basic TM principles, CHOP specific TM policies, as well as on the general processes/procedures of blood product testing and release. We, therefore, developed a teaching curriculum in 2006 to introduce anesthesiology fellows to the unique features of pediatric TM and hospital specific policies. In this study, we describe our experience with this curriculum over a four year period.

\section{Materials and methods}

A total of 28 fellows in the PACCM Training Program at CHOP, Philadelphia, rotated through the blood bank for a day. A brief tour of the blood bank was given followed by lectures on blood bank protocols and processes, the blood donor program and antibody identification. Hands-on demonstrations on ABO Rh typing, antibody screening, component preparation and modification and antibody panel identification were then given at the bench. Teaching materials

Table I Test questions. that were handed out included: lecture outlines, selected TM literature and blood bank information sheets (e.g. fast fact sheet for physicians obtaining informed consent for transfusion, minimum times for the blood bank processes, labeling of blood bank specimens and blood bank directed donor policy). A multiple-choice self-assessment questionnaire that consisted of four questions (Table 1) was given before (pre- test) and after (post-test) the rotation to assess learning. An evaluation of the rotation was also given at the end of the day (Table 2) to assess the perceived utility and effectiveness of the rotation. The evaluation utilized a scale from 1 (outstanding) to 5 (poor). The day ended with a wrap-up session with the blood bank medical directors.

The transfusion services laboratory client satisfaction survey is a biennial survey conducted by the blood bank to assess its performance and elicit feedback from hematology/oncology, surgical, and anesthesiology and critical care medicine department attending physicians on how to improve its services. This survey includes 10 questions on the quality of blood bank products and services as well as on the performance of the blood bank and its staff. Each question is rated on a scale of 1 (poor) to 5 (excellent). A comparison of survey results the year (2007) and 4 years (2010) after the implementation of the curriculum was performed by calculating the percent change in average score for each question from the 2007 survey. Results of the self-assessment questionnaires and evaluations are given as mean $\pm \mathrm{SD}$ or count. Statistical analyses were performed using Stata version 11. A two-tailed Student's t test was used to determine statistically significant differences between pre-and post-test results. A p-value less than 0.05 were considered statistically significant.

\begin{tabular}{|c|c|}
\hline Questions & Possible answers* \\
\hline & a. Antibodies that react at room temperature \\
\hline \multirow{4}{*}{$\begin{array}{l}\text { What is the blood bank definition of } \\
\text { clinically significant antibodies? }\end{array}$} & b. Antibodies that are passively acquired \\
\hline & c. Antibodies that can cause hemolytic disease of the newborn and/or hemolytic transfusion reactions \\
\hline & d. Antibodies that prewarm away and are compatible at cross matching \\
\hline & a. A negative or A positive \\
\hline \multirow{3}{*}{$\begin{array}{l}\text { What type of directed donor red } \\
\text { cells will be accepted for an A } \\
\text { positive patient according to CHOP } \\
\text { policy? }\end{array}$} & b. O negative or $O$ positive \\
\hline & d. B positive or A positive \\
\hline & a. Patient's name, date of birth and collectors initials \\
\hline \multirow{3}{*}{$\begin{array}{l}\text { What is the labeling criterion for a } \\
\text { sample to be accepted as a CHOP } \\
\text { Blood Bank sample? }\end{array}$} & b. Patient's first and last name, medical record number, date and collectors name \\
\hline & c. Patient's last name, first initial and account number \\
\hline & d. No labeling requirements \\
\hline & a. Wash 24 hours after irradiation, expires 2 days after washing \\
\hline \multirow{3}{*}{$\begin{array}{l}\text { When red cells are irradiated and } \\
\text { not used, when are the red cells } \\
\text { washed according to CHOP policy } \\
\text { and when do red cells expire after } \\
\text { washing? }\end{array}$} & b. Wash 5 days after irradiation, expires 8 hours after washing \\
\hline & c. Wash 3 days after irradiation, expires 3 days after washing \\
\hline & d. Wash 48 hours after irradiation, expires 24 hours after washing \\
\hline
\end{tabular}


Table 2 Rotation evaluation

\begin{tabular}{|c|c|c|c|c|c|c|c|}
\hline Category & $\begin{array}{l}\text { Outstanding } \\
\text { I }\end{array}$ & $\begin{array}{l}\text { Excellent } \\
2\end{array}$ & $\begin{array}{l}\text { Good } \\
3\end{array}$ & $\begin{array}{l}\text { Fair } \\
4\end{array}$ & $\begin{array}{l}\text { Poor } \\
5\end{array}$ & $\begin{array}{l}\text { Responses } \\
\text { (N) }\end{array}$ & $\begin{array}{l}\text { Average } \\
\text { Score } \pm S D\end{array}$ \\
\hline Clarity of Goals & $13(56.5 \%)$ & $9(39.1 \%)$ & $\mathrm{I}(4.3 \%)$ & 0 & 0 & 23 & $1.478 \pm 0.593$ \\
\hline Achievement of Goals & $13(56.5 \%)$ & $9(39.1 \%)$ & $\mathrm{I}(4.3 \%)$ & 0 & 0 & 23 & $1.478 \pm 0.593$ \\
\hline $\begin{array}{l}\text { Effectiveness of Lab } \\
\text { Demonstrations }\end{array}$ & II (50 \%) & II(50\%) & 0 & 0 & 0 & 22 & $1.500 \pm 0.512$ \\
\hline Effectiveness of Lectures & $10(43.5 \%)$ & $\mathrm{II}(47.8 \%)$ & $2(8.7 \%)$ & 0 & 0 & 23 & $1.652 \pm 0.647$ \\
\hline Effectiveness of Handouts & $12(57.1 \%)$ & $8(38.1 \%)$ & $\mathrm{I}(4.8 \%)$ & 0 & 0 & 21 & $1.476 \pm 0.602$ \\
\hline $\begin{array}{l}\text { Usefulness of Serology Case } \\
\text { Studies }\end{array}$ & $8(34.8 \%)$ & $10(43.5 \%)$ & $5(21.7 \%)$ & 0 & 0 & 23 & $1.863 \pm 0.774$ \\
\hline $\begin{array}{l}\text { Usefulness of Supplemental } \\
\text { Teaching Material }\end{array}$ & I I(55\%) & $9(45 \%)$ & 0 & 0 & 0 & 20 & $1.450 \pm 0.510$ \\
\hline Time Allocation & $14(60.9 \%)$ & $7(30.4 \%)$ & $2(8.7 \%)$ & 0 & 0 & 23 & $1.478 \pm 0.665$ \\
\hline Overall Rating & $13(59.1 \%)$ & 9 (40.9\%) & 0 & 0 & 0 & 22 & 1.4090 .503 \\
\hline
\end{tabular}

\section{Results}

Twenty-eight pre-and post-tests were returned. Out of 28 fellows, 27 answered question $1(0.964 \pm 0.189)$ correctly in the pre-and posttests. Twenty-five of 28 and 28 of 28 fellows answered question 3 (pre-test: $0.893 \pm 0.315$, post-test: $1 \pm 0$ ) correctly in the pre- and posttests, respectively. There was a statistically significant difference in pre-and post-test scores for question 2 (pre-test: $0.571 \pm 0.504$, posttest: $0.857 \pm 0.356 ; \mathrm{p}=0.02$ ) and question 4 (pre-test:0.679 \pm 0.476 , post-test: $1 \pm 0 ; \mathrm{p}<0.001)$ with the scores for these questions improving significantly in the post-test. Sixteen fellows answered A (A negative or A positive) and 11 fellows answered $\mathrm{C}$ (O positive or A positive) for question 2 in the pre-test. After the rotation, 24 out of 28 answered A which is the correct answer. For question 4, 19 fellows answered D (wash 48 hours after irradiation, expires 24hours after washing), 7 fellows answered A (wash 24hours after irradiation, expires 2days after washing) and 1 fellow answered B (wash 5days after irradiation, expires 8hours after washing) in the pre-test. After the rotation, all fellows answered D, which is the correct answer.

The evaluation form was filled out by 23 fellows (Table 2). The overall rating of the blood bank rotation was excellent $(n=9)$ to outstanding $(\mathrm{n}=13)(1.409 \pm 0.503)$. The goals and guidelines were thought to be clearly explained and achieved (1.478 \pm 0.593$)$. Eighteen out of 23 fellows participated in the laboratory demonstrations which were thought to be effective $(1.500 \pm 0.512)$. The lectures (1.652 \pm 0.647$)$, handouts $(1.476 \pm 0.602)$, serology case studies $(1.863 \pm 0.774)$ and supplemental teaching materials $(1.450 \pm 0.510)$ were thought to be useful and effective. The time allocated to this rotation was thought to be sufficient $(1.478 \pm 0.665)$. The level of material was thought to be just right by 22 out of 23 fellows (data not shown).

Fellows indicated that the strengths of the rotation included,

i. the lectures on blood product utilization and blood bank operations and policy;

ii. lecturers who were enthusiastic, organized and willing to answer questions;

iii. tour of the blood bank;

iv. laboratory demonstration; v. small group size; and

vi. Timing during their fellowship.

The perceived weaknesses included,

i. too much detail given regarding antibody identification using panel and select cells,

ii. insufficient time spent talking with the blood bank directors and

iii. The absence of a lecture on emergency/trauma blood bank protocols.

Topics that were suggested for future lectures in pediatric transfusion medicine included transfusion reactions, $\mathrm{ABO}$ incompatible transplantation, craniofacial surgery transfusion, infectious disease risks/bacterial transmission, factor concentrates, emergency/trauma blood bank protocols, component therapy and exchange transfusions. An increase in client satisfaction of transfusion laboratory services was observed between 2007 and 2010. Based on 19 responses for the 2010 survey and 29 responses for the 2007 survey, the overall satisfaction for the transfusion services laboratory increased by $18.3 \%$ (data not shown).

\section{Discussion}

We report the development of a TM curriculum designed to instruct a target group of end users on pediatric TM. This curriculum was designed based on learning needs for pediatric anesthesiology fellows. Two fellows rotated through the blood bank at a time over the course of a year. Our goal was to impart a basic understanding of key TM concepts as well as to educate the fellows on what the hospital policies were in order to improve patient safety, patient care and work flow. Questions used in the pre-and post-tests were designed to assess this knowledge of key concepts in TM as well as important $\mathrm{CHOP}$ specific policies. Fellows demonstrated an improvement in TM knowledge as well as CHOP specific policies after the rotation. Different hospitals have different policies regarding blood product processing, release and utilization. Thus, it is important for each institution to teach hospital specific policies to their fellows/residents.

Question two was the most frequent incorrectly answered question in the self-assessment questionnaire. This question addresses 
knowledge of CHOP policy regarding the type of directed donor red cells that will be acceptable for a patient. The concern and discussion about this policy through this educational effort has prompted a recent review of this practice and may result in a CHOP policy change allowing the use of compatible blood from directed donors. The policy regarding the type of directed donor red cells that will be acceptable for a patient in other hospitals, however, could be different. A recent survey of US and Canadian children's hospitals in 2008 and 2009 noted a wide range of RBC transfusion policies for both neonatal and pediatric populations. With the lack of standardized practices, knowledge of institutional practices becomes even more critical. ${ }^{7}$

This one-day curriculum has been well received by the fellows that have rotated through as indicated in the evaluation results. One full day in the blood bank is sufficient to give a brief overview of blood bank operations and allows minimal interruption of PACCM education. Several didactic lectures were scheduled throughout the day in order to maximize learning and minimize information overload. In order to reinforce knowledge, help improve concern over insufficient time with blood bank medical directors and build a collaborative relationship between anesthesiology fellows over time, TM lectures were also given as part of the anesthesiology department's weekly fellow teaching conferences which occurs three times in the first few months of fellowship training. The topics of these lectures included: The use of fresh whole blood and red cell components, neonatal and sickle cell anemia specific transfusion practices, and an anesthesiology approach to pediatric blood banking with a focus on blood product usage guidelines, transfusion reactions, and selected product modifications such as irradiation and leukoreduction. Additional lectures, such as computerized physician order entry, were identified as a need based on fellow feedback. These lectures were given to both the attending and fellow staff. The lectures in combination with the rotation generated discussion and a collaborative environment which has then resulted in performance improvement initiatives as well as translational research including the design of new programs, such as a matched RBC and FFP program for donor limitation, and the study of whole blood coagulation properties. A brief and focused educational program has been found by Soumerai et al. ${ }^{8}$ to substantially improve the appropriateness and cost-effectiveness of blood product use in surgery. They conducted a randomized, controlled multicenter trial with a 6-month follow-up to determine whether brief, face-to-face education outreach visits can improve the appropriateness of blood product utilization. Among surgeons, they found that the average proportion of transfusions not in compliance with criteria declined by $40 \%$ compared with an increase of $9 \%$ in the control group. They also found that these surgeons performed transfusions when hematocrits were 2.0 percentage points lower after the intervention than before the intervention and when compared to the control group.

Opportunities to develop novel methods of training, such as simulation-based learning, have also developed out of this curriculum. There are numerous benefits associated with simulation-based learning, ${ }^{9-11}$ the foremost being that it allows physicians to develop their knowledge, skills and attitudes without putting patients at risk. The realistic scenarios and equipment allow for retraining and practice until one can master the procedure or skill and it also allows the training of interdisciplinary medical teams. ${ }^{11}$ Furthermore, simulationbased training techniques, tools, and strategies are useful not only for designing structured learning experiences but also for providing a measurement tool linked to targeted teamwork competencies and learning objectives. ${ }^{11}$
Recent work at CHOP has focused on the design of simulation education centered on using uncrossmatched blood, in addition to ordering and issuing blood for trauma patients requiring transfer to the ORs. We have found opportunities for improvement in the use of the hospital order entry system as well as understanding the differences between a type and screen as well as a type and crossmatch (order to transfuse). The ability to observe this testing in the laboratory and then simulate the ordering of these tests in a "crisis simulated environment" prepares the fellows well for understanding their ordering requests for their patients. It also provides the opportunity to teach attending staff.

A transfusion services laboratory client satisfaction survey is sent out each year to the hematology/oncology, surgical, and anesthesiology and critical care medicine department attending physicians in order to evaluate the performance of the TM laboratory and staff. A modest increase in client satisfaction has been observed over the four years that the curriculum has been in place. Although causality between the curriculum and improved satisfaction cannot be objectively established, it can be hypothesized that at least part of the improved satisfaction with the blood bank stems from a better understanding of blood bank operations, policies and work flow which can be attributed in part to the new curriculum. Even though only a few attendings participated and it was primarily the fellows who were being directly educated through this curriculum, attending physicians could have better satisfaction with the blood bank because they were educated by the fellows and/or because of more efficient interactions between their trainees and the blood bank. We have also observed a decrease in our customer complaints and safety net reports resulting from miscommunication between the blood bank and critical care staff.

One of the didactic components that is required by the ACGME for pediatric anesthesiology fellows is on coagulation abnormalities and therapy. The TM curriculum that we have developed could satisfy a part of this requirement. It is important for anesthesiology and critical care medicine fellows who will be future attendings to have a good grasp of basic TM concepts and blood bank operations in order facilitate their work flow and improve patient safety. It is essential that anesthesiology faculty be well versed in the program requirements they must teach anesthesiology and critical care fellows.

An improvement in the teaching of TM should not only occur at the fellow level and not only for PACCM housestaff. Teaching basic TM concepts should start at the medical student level and should be reinforced throughout successive training as an intern, resident, fellow and even attending. O'Brien et al. ${ }^{3}$ reports that $34.5 \%$ of PGY1 's receive only a single lecture and $41.4 \%$ receive no training at all. In 1995, Cable et al. ${ }^{12}$ published a revised medical school curriculum that was approved by the Transfusion Medicine Academic Awards (TMAA) Group. The revised curriculum consists of 28 objectives in six major areas of transfusion medicine. Recently, the Pediatric TMAA group identified 65 objectives for a pediatric transfusion medicine curriculum, 23 of which are core objectives which should be considered for clinical pathology training. ${ }^{13}$ Educating attendings about TM may be warranted based on the results of a study by SalemSchatz SR in 19904 that found that attending physicians routinely had lower knowledge scores than did residents, but they were more confident in their knowledge. They found that residents' transfusion decisions were strongly influenced by the desires of their attending physicians, resulting in their ordering potentially inappropriate transfusions. ${ }^{4}$ As a result in the positive educational experience of our 
TM anesthesia fellow curriculum, the program was extended to our anesthesia resident training through a rotation in the blood bank at The Hospital of The University of Pennsylvania.

We recognize challenges in the current assessment of our curriculum as to the lack of long term follow-up from our trainees and our limited evaluation with the use of only four pre-and posttest questions. The pre- and post- test questions were designed as a simple assessment tool and could be more comprehensive in the future. Also, the names were not required on the pre- and post-tests or feedback form, but sometimes the fellows attached their name to the data. In this way, the process was not entirely anonymous. In addition, tracking blood utilization, performance improvement initiatives and customer satisfaction surveys and safety net reporting are only indirect evidence for improvements made as a result of the curriculum. This TM curriculum developed for a select end user group will continue to evolve as we work towards developing better tools for feedback and assessment of our educational efforts. Some of these tools in development include a survey to be conducted among the anesthesia and critical care attending staff to collect more precise feedback on the curriculum design, opportunities for future improvements, as well as progress as a result of the fellows' curriculum.

\section{Conclusion}

We have developed a TM curriculum PACCM fellows' to learn basic TM concepts, understand blood bank operations, and know CHOP specific policies regarding the use and processing of blood components. Fellows' knowledge of TM increased after participating in this rotation which was reflected in improved scores in the post-test as well as in improved overall client satisfaction with the transfusion services laboratory over the 4 year study period. This curriculum will continue to evolve as additional feedback is received on the relevance of the teaching to the practice of pediatric anesthesiology. The future development of similar educational programs which involve the ordering, testing, issuing and transfusion of blood components will enhance the training experience of the current CHOP fellows as well as influence practice-based knowledge in their future jobs and institutions. This is one way to encourage the growth of interest and training of future leaders in the specialty of pediatric TM. Because of the positive feedback that has been received from rotating fellows and residents who participate as an elective, we plan to continue this educational effort to offer this curriculum to other departments at CHOP.

\section{Acknowledgements}

None.

\section{Conflict of interest}

The author declares no conflict of interest.

\section{References}

1. Rock G, Berger R, Pinkerton P, et al. A pilot study to assess physician knowledge in transfusion medicine. Transfus Med. 2002;12(2):125-128.

2. Eisenstaedt RS, Glanz K, Polansky M. Resident education in transfusion medicine: a multi-institutional needs assessment. Transfusion. 1988;28(6):536-540.

3. O'Brien KL, Champeaux AL, Sundell ZE, et al. Transfusion medicine knowledge in postgraduate year 1 residents. Transfusion. 2010;50(8):1649-1653

4. Salem-Schatz SR, Avorn J, Soumerai SB. Influence of clinical knowledge, organizational context, and practice style on transfusion decision making. Implications for practice change strategies. JAMA. 1990;264(4):476-83.

5. Mitchell SA, Strauss RG, Albanese MA, et al. A survey to identify deficiencies in transfusion medicine education. Acad Med. 1989;64(4):217-229.

6. Bergmann H. Anaesthesiology and transfusion medicine. Infusionstherapie. 1991;18(1):12-19.

7. Spinella PC, Dressler A, Tucci M, et al. Survey of transfusion policies at US and Canadian children's hospitals in 2008 and 2009. Transfusion. 2010;50(11):2328-2335.

8. Soumerai SB, Salem-Schatz S, Avorn J, et al. A controlled trial of educational outreach to improve blood transfusion practice. JAMA. 1993;270(8):961-966.

9. Huang GC, Gordon JA, Schwartzstein RM. Millennium conference 2005 on medical simulation: a summary report. Simul Healthc. 2007;2(2):8895.

10. Huang YM, Pliego JF, Henrichs B, et al. 2007 simulation education summit. Simul Healthc. 2008;3(3):186-191.

11. Lateef F. Simulation-based learning: Just like the real thing. J Emerg Trauma Shock. 2010;3(4):348-352.

12. Cable RG, Thal SE, Fink A, et al. A comprehensive transfusion medicine curriculum for medical students. Transfusion Medicine academic award group. Transfusion. 1995;35(6):465-469.

13. Sanchez R, Sloan SR, Josephson CD, et al. Consensus recommendations of pediatric transfusion medicine objectives for clinical pathology residency training programs. Transfusion. 2010;50(5):1071-1078. 